\title{
Molecular Modeling Studies of Some Substituted 2-Phenyl-benzimidazole Derivatives as Inhibitors of IgE Response
}

\author{
Mukesh C. Sharma \\ Drug Research Laboratory, School of Pharmacy, Devi Ahilya University, Takshila Campus, Khandwa Road, Indore, M.P, India
}

*Corresponding author: Sharma MC, Drug Research Laboratory, School of Pharmacy, Devi Ahilya University, Takshila Campus, Khandwa Road, Indore, M.P, India, Tel: 91-731-216005; E-mail: mukeshcsharma@yahoo.com

Rec date: Mar 12, 2015; Acc date: Apr 11, 2015; Pub date: Apr15, 2015

Copyright: @ 2015 Sharma MC. This is an open-access article distributed under the terms of the Creative Commons Attribution License, which permits unrestricted use, distribution and reproduction in any medium, provided the original author and source are credited.

\begin{abstract}
We perform the two-dimensional (2D) QSAR studies of a series of substituted 2-phenyl-benzimidazole analogues to elucidate the structural properties required inhibitors of IgE response. The 2D-QSAR studies were performed using three statistical methods: the multiple linear regressions, giving square of correlation coefficient $r^{2}=0.8386$, cross validated squared correlation coefficient $\mathrm{q}^{2}=0.7218$ and predictable ability pred_ $\mathrm{r}^{2}=0.7525$; Multiple linear regression (MLR). The results show that the proposed 2D-QSAR models are valid and that they can be applied to predict the activities of substituted 2-phenyl-benzimidazole inhibitors of IgE response.
\end{abstract}

Keywords: 2D QSAR; Multiple linear regression; Benzimidazole analogues; IgE responses; Asthma

\section{Introduction}

Asthma is a chronic respiratory disease that affects 300 million adults and children worldwide, including 15.7 million adults and 6.5 million children in the United States [1]. The prevalence has increased by $50 \%$ in the past few decades, particularly in Westernized countries. Although corticosteroids and $\beta 2$-agonists are effective in managing asthma symptoms, there is no curative therapy. There are also concerns regarding the side effects from chronic use of current drugs, particularly by children. The chronic nature of asthma and the lack of preventive and curative therapy are leading patients with asthma in Western societies to seek complementary and alternative medicine (CAM) treatment [2,3]. Human allergic disorders (type I hypersensitivity responses) ranging from hay fever, excema, and food allergies to potentially life threatening asthma and anaphylactic shock are increasing worldwide [4]. Central to the cascade of events that lead to these clinical allergic manifestations are protein-protein binding events between human immunoglobulin $\mathrm{E}$ (hIgE) and its class specific Fc receptors on effector cells [5-7]. Allergic asthma is a multifactorial disease, influenced by genetic and environmental factors, and is characterized by bronchial hyperresponsiveness, the presence of $\operatorname{IgE}$ antibodies to inhalant allergens and often also by enhanced total serum IgE levels. A switch recombination of antibodies to IgE requires two signals from activated T cells: the expression of the ligand for CD40 and the secretion of IL- 4 or IL-13. Both IL-4 and IL-13, independently of each other, are able to induce IgE antibody [8-10]. The allergen-IgE interactions on the mast cell surface initiate a complex series of downstream signaling cascades, including phosphorylation of the immunoreceptor tyrosine-based activation motifs (ITAMs) in the $\beta$ - and $\gamma$-chains of FceRI, resulting in mast cell degranulation $[11,12]$. While there are a number of pharmacological agents available for the treatment of asthma and allergic rhinitis, a major shortcoming of many of these therapeutic alternatives is that they impact the disease state by targeting a single mediator that modifies a response at the target organ. By acting on effecter molecules, these drugs provide some symptomatic relief but do not modulate the course of the disease. Anti-histamines, for example, continue to be the drugs of choice for allergic rhinitis because they are somewhat effective and are linked to few side effects [13].

QSAR refers to a discipline in computational chemistry that addresses the modeling of biological activities or chemical reactivity based on the quantitative description for the chemical structure of molecules. QSAR relies on the basic assumption that molecules with similar physicochemical properties or structures will have similar activities [14].

Quantitative structure activity relationship (QSAR) is one of the most important areas in chemometrics, and is a valuable tool that is used extensively in drug design and medicinal chemistry. Once a reliable QSAR model is established, we can predict the activities of molecules, and know which structural features play an important role in biological processes [15]. Since the QSAR model and properties of molecules can be obtained based on descriptors, choosing the most relevant descriptors is necessary. Therefore, using a technique as variable selection for extent number of descriptors is the most essential step in QSAR study [16]. The present paper deals with the novel development of drugs for the category, substituted 2-phenylbenzimidazole derivatives inhibitors of IgE response.

The main purpose of quantitative structure-activity relationship (QSAR) analyses is to make activity predictions for unknown compounds to guide the structure-based design of new analogues. Multiple linear regression (MLR) models have been developed as a mathematical equation which can relate chemical structure to the activity. The results obtained will be helpful to pharmacologists, chemists and medicinal chemists to come up with improved IgE responses drugs.

\section{Materials and Method}

A dataset of ninety four 2-phenyl-benzimidazole derivatives [17] (Richards et al.) for their IgE response have been taken for present QSAR work given in Table 1. Total of ninety four molecules were 
Citation: Sharma MC (2015) Molecular Modeling Studies of Some Substituted 2-Phenyl-benzimidazole Derivatives as Inhibitors of IgE Response. Altern Integr Med 4: 191. doi:10.4172/2327-5162.1000191

Page 2 of 9

considered for this study out of which twenty four molecules were used as test set.

The test set was chosen so as to accommodate compounds with activities in a different range. QSAR models were developed for both the training and the test set molecules, and the test set was used to validate the developed models.

In $\log \mathrm{IC}_{50}$ values were converted to $-\log \mathrm{IC}_{50}$ in order to bring out better linear correlations and reduce clustering of compounds while generating QSAR regression lines. The experimental information associated with biological activity, which is used as dependent variables in building a QSAR model. In this study, computational work (2D-QSAR) was performed using Vlife MDS QSAR plus software [18] on a HP computer with Core2 Duo processor and a window XP operating system.

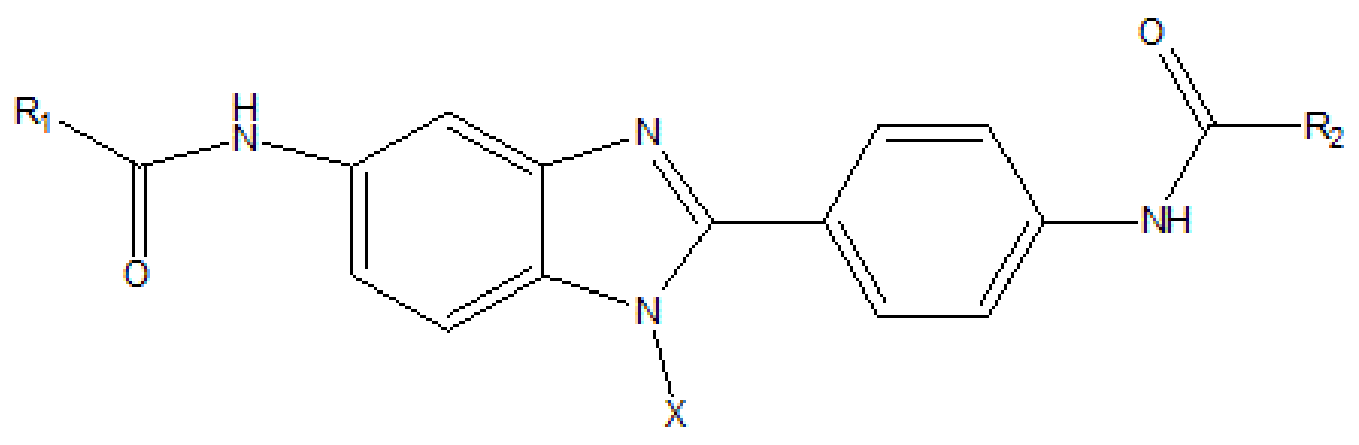

\begin{tabular}{|c|c|c|c|c|c|c|}
\hline S.No & R1 & R2 & $\mathbf{x}$ & $\mathbf{Y}$ & $\mathbf{I C}_{50}$ & $\mathrm{plC}_{50}$ \\
\hline 1 & Phenyl & Phenyl & $\mathrm{H}$ & $\mathrm{H}$ & 20 & 7.698 \\
\hline 2 & 4-Bromopheny & 4-Bromophenyl & $\mathrm{H}$ & $\mathrm{H}$ & 200 & 6.698 \\
\hline 3 & 3-Chlorophenyl & 3-Chloropheny & $\mathrm{H}$ & $\mathrm{H}$ & 25 & 7.602 \\
\hline 4 & 2-Chlorophenyl & 2-Chloropheny & $\mathrm{H}$ & $\mathrm{H}$ & 45 & 7.346 \\
\hline 5 & 3,4-Dichlorophenyl & 3,4-Dichlorophenyl & $\mathrm{H}$ & $\mathrm{H}$ & 40 & 7.397 \\
\hline $6^{*}$ & 2,3-Dichlorophenyl & 2,3-Dichlorophenyl & $\mathrm{H}$ & $\mathrm{H}$ & 10 & 8.000 \\
\hline 7 & 3,5-Dichlorophenyl & 3,5-Dichlorophenyl & $\mathrm{H}$ & $\mathrm{H}$ & 70 & 7.154 \\
\hline 8 & 2,4-Dichlorophenyl & 2,4-Dichlorophenyl & $\mathrm{H}$ & $\mathrm{H}$ & 30 & 7.522 \\
\hline 9 & 2,6-Dichlorophenyl & 2,6-Dichlorophenyl & $\mathrm{H}$ & $\mathrm{H}$ & 400 & 6.397 \\
\hline $10^{*}$ & Penta-fluoro-phenyl & Penta-fluoro-phenyl & $\mathrm{H}$ & $\mathrm{H}$ & 4 & 8.397 \\
\hline 11 & Phenyl & 4-Chlorophenyl & $\mathrm{H}$ & $\mathrm{H}$ & 90 & 7.045 \\
\hline 12 & 4-Nitrophenyl & 4-Nitrophenyl & $\mathrm{H}$ & $\mathrm{H}$ & 150 & 6.823 \\
\hline $13^{*}$ & 4-Cyanophenyl & 4-Cyanophenyl & $\mathrm{H}$ & $\mathrm{H}$ & 100 & 7 \\
\hline 14 & 4-Methoxyphenyl & 4-Methoxyphenyl & $\mathrm{H}$ & $\mathrm{H}$ & 30 & 7.522 \\
\hline 15 & 3,4-Dimethoxyphenyl & 3,4-Dimethoxyphenyl & $\mathrm{H}$ & $\mathrm{H}$ & 700 & 6.154 \\
\hline 16 & 4-S-methyl-phenyl & 4-S-methyl-phenyl & $\mathrm{H}$ & $\mathrm{H}$ & 150 & 6.823 \\
\hline $17^{*}$ & 4-Methylphenyl & 4-Methylphenyl & $\mathrm{H}$ & $\mathrm{H}$ & 20 & 7.698 \\
\hline 18 & 1-Naphthalene & 1-Naphthalene & $\mathrm{H}$ & $\mathrm{H}$ & 80 & 7.096 \\
\hline 19 & $\mathrm{CH} 2$-2-thiophene & $\mathrm{CH} 2-2$-thiophene & $\mathrm{H}$ & $\mathrm{H}$ & 500 & 6.301 \\
\hline 20 & Cyclohex-3-ene & Cyclohex-3-ene & $\mathrm{H}$ & $\mathrm{H}$ & 400 & 6.397 \\
\hline
\end{tabular}


Citation: Sharma MC (2015) Molecular Modeling Studies of Some Substituted 2-Phenyl-benzimidazole Derivatives as Inhibitors of IgE Response. Altern Integr Med 4: 191. doi:10.4172/2327-5162.1000191

Page 3 of 9

\begin{tabular}{|c|c|c|c|c|c|c|}
\hline $21^{*}$ & Phenyl- & Cyclohexyl & $\mathrm{H}$ & $\mathrm{H}$ & 10 & 8 \\
\hline 22 & $\mathrm{CH} 3$ & Cyclohexyl & $\mathrm{H}$ & $\mathrm{H}$ & 100 & 7 \\
\hline 23 & 3,4-Dichlorophenyl & Cyclohexyl & $\mathrm{H}$ & $\mathrm{H}$ & 0.8 & 9.096 \\
\hline 24 & 4-Chlorophenyl & Cyclohexyl & $\mathrm{H}$ & $\mathrm{H}$ & 6 & 8.221 \\
\hline $25^{*}$ & Cyclohexyl & 3,4-Dichlorophenyl & $\mathrm{H}$ & $\mathrm{H}$ & 0.4 & 9.397 \\
\hline 26 & Cyclohexyl & 4-Chlorophenyl & $\mathrm{H}$ & $\mathrm{H}$ & 8.0 & 8.096 \\
\hline 27 & 1-Adamanty & 2-Fluorophenyl & $\mathrm{H}$ & $\mathrm{H}$ & 10 & 8 \\
\hline 28 & 1-Adamanty & 4-Fluorophenyl & $\mathrm{H}$ & $\mathrm{H}$ & 10 & 8 \\
\hline 29 & 2-Pyridyl & 1-Adamanty & $\mathrm{H}$ & $\mathrm{H}$ & 6 & 8.221 \\
\hline $30^{*}$ & 3-Pyridyl & 1-Adamanty & $\mathrm{H}$ & $\mathrm{H}$ & 20 & 7.698 \\
\hline 31 & Cyclohexyl & Cyclohexyl & $\mathrm{H}$ & $\mathrm{H}$ & 4 & 8.397 \\
\hline 32 & 1-Adamantyl & 1-Adamantyl & $\mathrm{H}$ & $\mathrm{H}$ & 4 & 8.397 \\
\hline 33 & Cycloheptyl & Cycloheptyl & $\mathrm{H}$ & $\mathrm{H}$ & 1.5 & 8.823 \\
\hline $34^{*}$ & Cyclobutyl & Cyclobutyl & $\mathrm{H}$ & $\mathrm{H}$ & 400 & 6.397 \\
\hline 35 & Cyclopropyl & Cyclopropyl & $\mathrm{H}$ & $\mathrm{H}$ & 1000 & 6 \\
\hline 36 & 4-Methyl-cyclohexyl & 4-Methyl-cyclohexyl & $\mathrm{H}$ & $\mathrm{H}$ & 4 & 8.397 \\
\hline 37 & Cinnamyl & Cinnamyl & $\mathrm{H}$ & $\mathrm{H}$ & 70 & 7.154 \\
\hline $38^{*}$ & Phenyl & Phenyl & $\mathrm{CH}_{3}$ & $\mathrm{H}$ & 800 & 6.096 \\
\hline 39 & Cyclohexyl & Cyclohexyl & $\mathrm{COOCH}_{2} \mathrm{CH}_{3}$ & $\mathrm{H}$ & 7 & 8.154 \\
\hline 40 & Cyclohexyl & Cyclohexyl & $\mathrm{COCH}_{3}$ & $\mathrm{H}$ & 1.5 & 8.823 \\
\hline 41 & Cyclohexyl & Cyclohexyl & $\mathrm{H}$ & $\mathrm{H}$ & 2 & 8.698 \\
\hline $42^{*}$ & 4-Methyl-phenyl & 4-Methyl-phenyl & $\mathrm{H}$ & $\mathrm{H}$ & 40 & 7.397 \\
\hline 43 & 4-Fluorophenyl & 4-Fluorophenyl & $\mathrm{H}$ & $\mathrm{H}$ & 150 & 6.823 \\
\hline 44 & 4-Methoxyphenyl & 4-Methoxyphenyl & $\mathrm{H}$ & $\mathrm{H}$ & 100 & 7 \\
\hline 45 & Phenyl & 3,4-Dichlorophenyl & $\mathrm{H}$ & $\mathrm{H}$ & 100 & 7 \\
\hline $46^{*}$ & Phenyl & 5-Methyl-2-pyridyl & $\mathrm{H}$ & $\mathrm{H}$ & 300 & 6.522 \\
\hline 47 & Cyclohexyl & Phenyl & $\mathrm{H}$ & $\mathrm{H}$ & 9 & 8.045 \\
\hline 48 & 1-Adamantyl & Phenyl & $\mathrm{H}$ & $\mathrm{H}$ & 25 & 7.602 \\
\hline 49 & Phenyl & 1-Adamantyl & $\mathrm{H}$ & $\mathrm{H}$ & 8 & 8.096 \\
\hline $50^{*}$ & 1-Adamantyl & 4-Chlorophenyl & $\mathrm{H}$ & $\mathrm{H}$ & 9 & 8.045 \\
\hline 51 & 1-Adamantyl & 3,4-Dichlorophenyl & $\mathrm{H}$ & $\mathrm{H}$ & 1.5 & 8.823 \\
\hline 52 & 2-Adamantyl & 3,4-Dichlorophenyl & $\mathrm{H}$ & $\mathrm{H}$ & 16 & 7.795 \\
\hline 53 & Cyclohexyl & 4-Fluorophenyl & $\mathrm{H}$ & $\mathrm{H}$ & 5 & 8.301 \\
\hline 54 & Cyclohexyl & 4-Chlorophenyl & $\mathrm{H}$ & $\mathrm{H}$ & 3 & 8.522 \\
\hline $55^{*}$ & 2-Adamantyl & 4-Methoxyphenyl & $\mathrm{H}$ & $\mathrm{H}$ & 7 & 8.154 \\
\hline 56 & 4-Methoxyphenyl & 1-Adamantyl & $\mathrm{H}$ & $\mathrm{H}$ & 40 & 7.397 \\
\hline
\end{tabular}


Citation: Sharma MC (2015) Molecular Modeling Studies of Some Substituted 2-Phenyl-benzimidazole Derivatives as Inhibitors of IgE Response. Altern Integr Med 4: 191. doi:10.4172/2327-5162.1000191

Page 4 of 9

\begin{tabular}{|c|c|c|c|c|c|c|}
\hline 57 & 4-Fluorophenyl & 2-Adamantyl & $\mathrm{H}$ & $\mathrm{H}$ & 40 & 7.397 \\
\hline $58^{*}$ & 1-Adamantyl & 2-Pyridyl & $\mathrm{H}$ & $\mathrm{H}$ & 10 & 8 \\
\hline 59 & 2-Adamantyl & 2-Pyridyl & $\mathrm{H}$ & $\mathrm{H}$ & 10 & 8 \\
\hline 60 & 2-Adamantyl & 3-Pyridyl & $\mathrm{H}$ & $\mathrm{H}$ & 20 & 7.698 \\
\hline $61^{*}$ & 2-Adamantyl & 4-Pyridyl & $\mathrm{H}$ & $\mathrm{H}$ & 40 & 7.397 \\
\hline 62 & 2-Pyridyl & 2-Adamantyl & $\mathrm{H}$ & $\mathrm{H}$ & 40 & 7.397 \\
\hline 63 & 2-Pyridyl & 1-Adamantyl & $\mathrm{H}$ & $\mathrm{H}$ & 40 & 7.397 \\
\hline 64 & 2-Adamantyl & 5-Methyl-2-pyridyl & $\mathrm{H}$ & $\mathrm{H}$ & 20 & 7.698 \\
\hline $65^{*}$ & Cyclohexyl & Cyclohexyl & $\mathrm{H}$ & $\mathrm{H}$ & 80 & 7.096 \\
\hline 66 & 1-Adamantyl & 1-Adamantyl & $\mathrm{H}$ & $\mathrm{H}$ & 16 & 7.795 \\
\hline 67 & 4-Methyl-cyclohexyl & 4-Methyl-cyclohexyl & $\mathrm{H}$ & $\mathrm{H}$ & 35 & 7.455 \\
\hline 68 & 1-Adamantyl & Cyclohexyl & $\mathrm{H}$ & $\mathrm{H}$ & 8 & 8.096 \\
\hline $69^{*}$ & 2-Adamantyl & 2-Methyl-cyclohexyl & $\mathrm{H}$ & $\mathrm{H}$ & 4 & 8.397 \\
\hline 70 & 2-Methyl-cyclohexyl & 1-Adamantyl & $\mathrm{H}$ & $\mathrm{H}$ & 4 & 8.397 \\
\hline 72 & Phenyl & Phenyl & $\mathrm{H}$ & $\mathrm{H}$ & 400 & 6.397 \\
\hline 72 & 3,4-Dichlorophenyl & Phenyl & $\mathrm{H}$ & $\mathrm{H}$ & 50 & 7.301 \\
\hline $73^{*}$ & Phenyl & Cyclohexyl & $\mathrm{H}$ & $\mathrm{H}$ & 70 & 7.154 \\
\hline 74 & Cyclohexyl & Phenyl & $\mathrm{H}$ & $\mathrm{H}$ & 130 & 6.886 \\
\hline 75 & 1-Adamantyl & Phenyl & $\mathrm{H}$ & $\mathrm{H}$ & 150 & 6.823 \\
\hline $76^{*}$ & Phenyl & 1-Adamantyl & $\mathrm{H}$ & $\mathrm{H}$ & 4 & 8.397 \\
\hline 77 & 3,4-Dichlorophenyl & 1-Adamantyl & $\mathrm{H}$ & $\mathrm{H}$ & 0.7 & 9.154 \\
\hline 78 & 3,4-Dichlorophenyl & Bicycloheptyl & $\mathrm{H}$ & $\mathrm{H}$ & 40 & 7.397 \\
\hline $79^{*}$ & 3,4-Dichlorophenyl & Cyclohexyl & $\mathrm{H}$ & $\mathrm{H}$ & 15 & 7.823 \\
\hline 80 & Cyclohexyl & Cyclohexyl & $\mathrm{H}$ & $\mathrm{H}$ & 50 & 7.301 \\
\hline 81 & 1-Adamantyl & 1-Adamantyl & $\mathrm{H}$ & $\mathrm{H}$ & 6 & 8.221 \\
\hline 82 & Cycloheptyl & Cycloheptyl & $\mathrm{H}$ & $\mathrm{H}$ & 3 & 8.522 \\
\hline 83 & Cycloheptyl & Cycloheptyl & $\mathrm{H}$ & $\mathrm{H}$ & 500 & 6.301 \\
\hline $84^{*}$ & Bicycloheptyl & Bicycloheptyl & $\mathrm{H}$ & $\mathrm{H}$ & 30 & 7.522 \\
\hline 85 & 2-Methyl-cyclohexyl & 2-Methyl-cyclohexyl & $\mathrm{H}$ & $\mathrm{H}$ & 60 & 7.221 \\
\hline 86 & 4-Methyl-cyclohexyl & 4-Methyl-cyclohexyl & $\mathrm{H}$ & $\mathrm{H}$ & 3 & 8.522 \\
\hline 87 & 1-Adamantyl & Cyclohexyl & $\mathrm{H}$ & $\mathrm{H}$ & 60 & 7.221 \\
\hline $88^{*}$ & Cyclohexyl & 1-Adamantyl & $\mathrm{H}$ & $\mathrm{H}$ & 4 & 8.397 \\
\hline 89 & 4-Methyl-cyclohexyl & 1-Adamantyl & $\mathrm{H}$ & $\mathrm{H}$ & 3 & 8.5228 \\
\hline 90 & Cyclohexyl & Bicycloheptyl & $\mathrm{H}$ & $\mathrm{H}$ & 30 & 7.522 \\
\hline $91^{*}$ & 1-Adamantyl & Bicycloheptyl & $\mathrm{H}$ & $\mathrm{H}$ & 20 & 7.698 \\
\hline 92 & 1-Adamantyl & Cycloheptyl & $\mathrm{H}$ & $\mathrm{H}$ & 3 & 8.522 \\
\hline
\end{tabular}


Page 5 of 9

\begin{tabular}{|l|l|l|l|l|l|l|}
\hline 93 & 1-Adamantyl & Cycloheptyl & H & H & 70 & 7.154 \\
\hline 94 & 2-Pyridyl & 1-Adamantyl & H & H & 40 & 7.397 \\
\hline "Test compound
\end{tabular}

Table 1: Structures and activities of benzimidazole derivatives as inhibitors of IgE response.

All the 2D descriptors were calculated for QSAR analysis using Vlife MDS 3.5 software. Energy minimization and geometry optimization were conducted using Merck molecular force field as force field and charge, maximum number of cycles were 1,000, convergence criterion (RMS gradient) was 0.01 and medium's dielectric constant of 1 by batch energy minimization method. The dataset of 94 molecules was divided into training set (71 compounds) and test set (23 compounds) by Sphere Exclusion (SE) method [19] for multiple linear regression (MLR) model with dissimilarity value of 11.2 using pEC50 activity field as dependent variable and various $2 \mathrm{D}$ descriptors as independent variables.

Energy-minimized geometry was used for calculation of descriptors, a total of 208 2D descriptors were calculated which encoded different aspects of molecular structure and consists of electronic, thermodynamic, spatial, and structural descriptors, e.g., retention index (chi), atomic valence connectivity index (chiV), path count, chain path count, cluster, path cluster, element count, estate number, semi-empirical, molecular weight, molecular refractivity, $\log \mathrm{P}$, and topological index.

\section{Multiple linear regression (MLR)}

MLR is a method used for modeling linear relationship between a dependent variable $\mathrm{Y}\left(\mathrm{pIC}_{50}\right)$ and independent variable $\mathrm{X}(2 \mathrm{D}$ descriptors). MLR is based on least squares: the model is fit such that sum-of-squares of differences of observed and a predicted value is minimized. MLR estimates values of regression coefficients $\left(\mathrm{r}^{2}\right)$ by applying least squares curve fitting method. The model creates a relationship in the form of a straight line (linear) that best approximates all the individual data points. In regression analysis, conditional mean of dependant variable (pEC50) Y depends on (descriptors) X. MLR analysis extends this idea to include more than one independent variable. Regression equation takes the form

$\mathrm{Y}=\mathrm{b} 1{ }^{*} \mathrm{x} 1+\mathrm{b} 2{ }^{*} \mathrm{x} 2+\mathrm{b} 3{ }^{*} \mathrm{x} 3+\mathrm{c}$

Where $\mathrm{Y}$ is dependent variable, 'b's are regression coefficients for corresponding ' $x$ 's (independent variable), 'c' is a regression constant or intercept $[20,21]$.

\section{Results and Discussion}

For QSAR analysis regression was performed using $\mathrm{pIC}_{50}$ values as dependent variables and calculated parameters as independent variables. In any thorough investigation of the effects of molecular properties, it is essential to prove that the results are both statistically valid. $2 \mathrm{D}$-QSAR equations were selected by optimizing the statistical results generated along with variation of the descriptors in this model.

$\mathrm{pIC}_{50}=0.3821( \pm 0.0259)$

SsOHE-index $+0.1694( \pm 0.0117)$

ChiV3Cluster $+0.4181( \pm 0.0356) \quad$ SssCH2E-index $-0.2025( \pm 0.0011)$

T_C_F_2+ 0.3689 $( \pm 0.0770)$ SsClE-index
Ntraining $=71$, Ntest $=23, \mathrm{r}^{2}=0.8386, \mathrm{q}^{2}=0.7218, \mathrm{~F}$ test $=43.3584$, $\mathrm{r}^{2} \_\mathrm{se}=0.1236, \mathrm{q}^{2}$ _ se $=0.1733$, pred_r ${ }^{2}=0.7525$, pred_r ${ }^{2} \mathrm{se}=0.4268$.

In above QSAR models, $\mathrm{r}^{2}$ is a correlation coefficient that has been multiplied by 100 gives explained variance in biological activity. Predictive ability of generated QSAR models was evaluated by $\mathrm{q}^{2}$ employing LOO method. F value reflects ratio of variance explained by models and variance due to error in regression. High $\mathrm{F}$ value indicates that model is statistically significant. Eq. (1) shows $84 \%$ variance in the observed activity values. The low standard error of $\mathrm{r}^{2} \_\mathrm{se}=0.1236$ demonstrates accuracy of the model.

Cross validated $\mathrm{q}^{2}$ of this model 0.7218 , indicates good internal prediction power of the model. Another parameter for predictivity of test set compounds is high pred_ $r^{2}=0.7525$, which shows good external predictive power of the model. The electro-topological parameter SsClcount define the total number of chlorine atoms connected with one single bond. The positive coefficient of the descriptor suggests that IgE responses of Substituted 2-phenyl-benzimidazole may be increased by increasing the number of chlorine atoms present in the nucleus.

The positive coefficient of the molecular connectivity index descriptor in the model suggests that the decrease in branching in the molecule and the presence of heteroatoms increases the IgE responses. An estate contribution electro-topological state descriptor SssCH2Eindex, which represents the indices for number of $-\mathrm{CH}_{2}$ group connected with two single bonds, is inversely proportional to the activity. The positive coefficient of T_C_F_2 in the QSAR model reveal that the presence of [fluoro-phenyl at the end terminal of benzimidazole increases activity. The contribution charts of selected descriptors are represented in Figure 1a. The Observed activity and Predicted activity pIC $_{50}$ along with residual values are shown in Table 2 and plots of observed vs. predicted values of $\mathrm{pIC}_{50}$ are shown in Figure 1b.

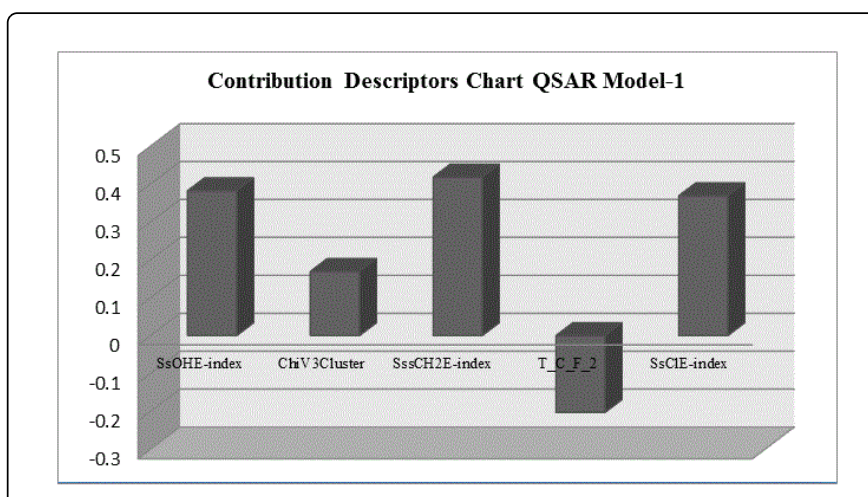

Figure 1a: Plot of contribution chart of 2D QSAR Model. 
Citation: Sharma MC (2015) Molecular Modeling Studies of Some Substituted 2-Phenyl-benzimidazole Derivatives as Inhibitors of IgE Response. Altern Integr Med 4: 191. doi:10.4172/2327-5162.1000191

Page 6 of 9

\begin{tabular}{|c|c|c|c|}
\hline \multirow{2}{*}{ Com } & \multirow{2}{*}{$\mathbf{p l C}_{50}$} & \multicolumn{2}{|c|}{ 2D-QSAR Model-1 } \\
\hline & & Pred. & Res. \\
\hline 1 & 7.698 & 7.5014 & 0.1966 \\
\hline 2 & 6.698 & 6.1844 & 0.5136 \\
\hline 3 & 7.602 & 7.1742 & 0.4278 \\
\hline 4 & 7.346 & 7.1565 & 0.1895 \\
\hline 5 & 7.397 & 7.2394 & 0.1576 \\
\hline $6^{*}$ & 8 & 8.1913 & -0.1913 \\
\hline 7 & 7.154 & 7.1385 & 0.0155 \\
\hline 8 & 7.522 & 7.3699 & 0.1521 \\
\hline 9 & 6.397 & 6.317 & 0.08 \\
\hline $10^{*}$ & 8.397 & 8.3898 & 0.0072 \\
\hline 11 & 7.045 & 7.3238 & -0.2788 \\
\hline 12 & 6.823 & 6.38 & 0.443 \\
\hline $13^{*}$ & 7 & 6.9905 & 0.0095 \\
\hline 14 & 7.522 & 7.3895 & 0.1325 \\
\hline 15 & 6.154 & 6.3705 & -0.2165 \\
\hline 16 & 6.823 & 6.3512 & 0.4718 \\
\hline $17^{*}$ & 7.698 & 7.349 & 0.349 \\
\hline 18 & 7.096 & 7.4522 & -0.3562 \\
\hline 19 & 6.301 & 6.308 & -0.007 \\
\hline 20 & 6.397 & 6.2796 & 0.1174 \\
\hline $21^{*}$ & 8 & 8.2619 & -0.2619 \\
\hline 22 & 7 & 7.3976 & -0.3976 \\
\hline 23 & 9.096 & 9.3444 & -0.2484 \\
\hline 24 & 8.221 & 8.4161 & -0.1951 \\
\hline $25^{*}$ & 9.397 & 9.3997 & -0.0027 \\
\hline 26 & 8.096 & 8.445 & -0.349 \\
\hline 27 & 8 & 8.378 & -0.378 \\
\hline 28 & 8 & 8.3608 & -0.3608 \\
\hline 29 & 8.221 & 8.395 & -0.174 \\
\hline $30^{*}$ & 7.698 & 7.3062 & 0.3918 \\
\hline 31 & 8.397 & 8.3479 & 0.0491 \\
\hline 32 & 8.397 & 8.2606 & 0.1364 \\
\hline 33 & 8.823 & 8.3629 & 0.4601 \\
\hline $34^{*}$ & 6.397 & 6.3113 & 0.0857 \\
\hline
\end{tabular}


Citation: Sharma MC (2015) Molecular Modeling Studies of Some Substituted 2-Phenyl-benzimidazole Derivatives as Inhibitors of IgE Response. Altern Integr Med 4: 191. doi:10.4172/2327-5162.1000191

Page 7 of 9

\begin{tabular}{|c|c|c|c|}
\hline 35 & 6 & 6.2892 & -0.2892 \\
\hline 36 & 8.397 & 8.2849 & 0.1121 \\
\hline 37 & 7.154 & 7.1138 & 0.0402 \\
\hline $38^{*}$ & 6.096 & 6.1735 & -0.0775 \\
\hline 39 & 8.154 & 8.1753 & -0.0213 \\
\hline 40 & 8.823 & 8.1666 & 0.6564 \\
\hline 41 & 8.698 & 8.2351 & 0.4629 \\
\hline $42^{*}$ & 7.397 & 7.1904 & 0.2066 \\
\hline 43 & 6.823 & 6.1705 & 0.6525 \\
\hline 44 & 7 & 7.3229 & -0.3229 \\
\hline 45 & 7 & 7.3268 & -0.3268 \\
\hline $46^{*}$ & 6.522 & 6.3362 & 0.1858 \\
\hline 47 & 8.045 & 8.2932 & -0.2482 \\
\hline 48 & 7.602 & 7.3312 & 0.2708 \\
\hline 49 & 8.096 & 8.3279 & -0.2319 \\
\hline $50^{*}$ & 8.045 & 8.3315 & -0.2865 \\
\hline 51 & 8.823 & 8.3254 & 0.4976 \\
\hline 52 & 7.795 & 7.2976 & 0.4974 \\
\hline 53 & 8.301 & 8.3018 & -0.0008 \\
\hline 54 & 8.522 & 8.364 & 0.158 \\
\hline $55^{*}$ & 8.154 & 8.2889 & -0.1349 \\
\hline 56 & 7.397 & 7.2641 & 0.1329 \\
\hline 57 & 7.397 & 7.2266 & 0.1704 \\
\hline $58^{*}$ & 8 & 8.3328 & -0.3328 \\
\hline 59 & 8 & 8.3162 & -0.3162 \\
\hline 60 & 7.698 & 7.3431 & 0.3549 \\
\hline $61^{*}$ & 7.397 & 7.3523 & 0.0447 \\
\hline 62 & 7.397 & 7.3951 & 0.0019 \\
\hline 63 & 7.397 & 7.3285 & 0.0685 \\
\hline 64 & 7.698 & 7.3244 & 0.3736 \\
\hline $65^{*}$ & 7.096 & 7.336 & -0.24 \\
\hline 66 & 7.795 & 7.2644 & 0.5306 \\
\hline 67 & 7.455 & 7.2963 & 0.1587 \\
\hline 68 & 8.096 & 8.2371 & -0.1411 \\
\hline $69^{*}$ & 8.397 & 8.278 & 0.119 \\
\hline 70 & 8.397 & 8.2609 & 0.1361 \\
\hline
\end{tabular}


Citation: Sharma MC (2015) Molecular Modeling Studies of Some Substituted 2-Phenyl-benzimidazole Derivatives as Inhibitors of IgE Response. Altern Integr Med 4: 191. doi:10.4172/2327-5162.1000191

Page 8 of 9

\begin{tabular}{|c|c|c|c|}
\hline 72 & 6.397 & 6.242 & 0.155 \\
\hline 72 & 7.301 & 7.238 & 0.063 \\
\hline $73^{*}$ & 7.154 & 7.093 & 0.061 \\
\hline 74 & 6.886 & 6.9321 & -0.0461 \\
\hline 75 & 6.823 & 6.4323 & 0.3907 \\
\hline $76^{*}$ & 8.397 & 8.3511 & 0.0459 \\
\hline 77 & 9.154 & 9.3763 & -0.2223 \\
\hline 78 & 7.397 & 7.4799 & -0.0829 \\
\hline $79^{*}$ & 7.823 & 7.4276 & 0.3954 \\
\hline 80 & 7.301 & 7.4261 & -0.1251 \\
\hline 81 & 8.221 & 8.4405 & -0.2195 \\
\hline 82 & 8.522 & 8.4509 & 0.0711 \\
\hline 83 & 6.301 & 6.4649 & -0.1639 \\
\hline $84^{*}$ & 7.522 & 7.4487 & 0.0733 \\
\hline 85 & 7.221 & 7.4676 & -0.2466 \\
\hline 86 & 8.522 & 8.5002 & 0.0218 \\
\hline 87 & 7.221 & 7.4985 & -0.2775 \\
\hline $88^{*}$ & 8.397 & 8.4923 & -0.0953 \\
\hline 89 & 8.522 & 8.4921 & 0.0299 \\
\hline 90 & 7.522 & 7.5365 & -0.0145 \\
\hline $91^{*}$ & 7.698 & 7.571 & 0.127 \\
\hline 92 & 8.522 & 8.3909 & 0.1311 \\
\hline 93 & 7.154 & 7.4208 & -0.2668 \\
\hline 94 & 7.397 & 7.4738 & -0.0768 \\
\hline
\end{tabular}

Table 2: Predicted activities according to 2D QSAR models results of benzimidazole with bacterial strains.

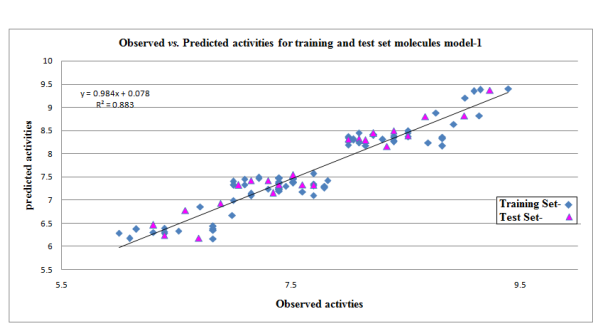

Figure 1b: Graphs of observed vs. predicted activity of 2D QSAR model-1.

\section{Conclusion}

A multiple linear regression (MLR) procedure was used to model the relationships between molecular descriptors and the inhibitors of IgE response of the benzimidazole derivatives. The quantitative structure-activity relationship (QSAR) analysis of some synthesized substituted 2-phenyl-benzimidazole derivatives inhibitors of $\operatorname{IgE}$ response were performed to find out the structural requirements of their IgE responses activities. Various 2D descriptors were calculated and used in the present analysis. The knowledge of Structure-Activity Relationship (SAR), together with the generation of QSAR, constitutes a large body of evidence that may assist in the development of new molecules with excellent biological activity and low toxicity. The results obtained from present investigation of IgE responses studies indicate that the presence of a chloropheny, fluoro-phenyl substituent leads to increase in the activity in comparison to the presence of a methyl group. QSAR analysis has been used to study the quantitative 
Citation: Sharma MC (2015) Molecular Modeling Studies of Some Substituted 2-Phenyl-benzimidazole Derivatives as Inhibitors of IgE Response. Altern Integr Med 4: 191. doi:10.4172/2327-5162.1000191

Page 9 of 9

effects of the molecular structure of the benzimidazoles on their inhibitory activity. In this model special emphasis was given to the contribution of electrotopological in predicting biological activity of 2phenyl-benzimidazole derivatives and they were found to improve the QSAR model and make it more precisely predictive.

\section{Acknowledgments}

The author wishes to express gratitude to V-life Science Technologies Pvt. Ltd for providing the trail version software for the study. Authors are thankful to the University Grants Commission (UGC), New Delhi for awarding a Research Award No. F. 30-1/2013(SA-II)/RA-2012-14-NEW-GE-MAD-4200.

\section{References}

1. http://www.cdc.gov/nchs/fastats/asthma.htm.

2. Hassed C (2005) An integrative approach to asthma. Australian Family Physician 34: 573-576.

3. Bielory L, Russin J, Zuckerman GB (2004) Clinical efficacy, mechanisms of action, and adverse effects of complementary and alternative medicine therapies for asthma. Allergy and Asthma Proceedings 25: 283-291.

4. Holgate ST (1999) The epidemic of allergy and asthma. Nature 402: 2-4.

5. Turner H, Kinet JP (1999) Signalling through the high-affinity IgE receptor Fc epsilon RI. Nature 402: 24-30.

6. Sutton B J, Gould H J (1993) The human IgE network. Nature 366: 421-428.

7. Sayers I, Helm BA (1999) the structural basis of human IgE-Fc receptor interactions. Clinical \& Experimental Allergy 29: 585-594.

8. Punnonen J, Aversa G, Cocks BG, McKenzie AN, Menon S, et al. (1999) Interleukin 13 induces interleukin 4-independent IgG4 and IgE synthesis and CD23 expression by human B cells. Proceedings of the National Academy of Sciences USA 90: 3730-3734.

9. Cocks BG, Malefyt R, Galizzi JP, Vries JE, Aversa G (1993) IL-13 induces proliferation and differentiation of human B cells activated by the CD40 ligand. International Immunology 5: 657-663.
10. Defrance T, Carayon P, Billian G, Guillemot JC, Minty A, et al. (1994) Interleukin 13 is a $\mathrm{B}$ cell stimulating factor. Journal of Experimental Medicine 179: 135-143.

11. Blank U, Rivera J (2006) Assays for regulated exocytosis of mast cell granules. Current Protocols in Cell Biology 15: 11.

12. Andrews NL, Pfeiffer JR, Martinez AM, Haaland DM, Davis RW, et al. (2009) Mobile Fc Epsilon R1 receptor aggregates are signaling competent. Immunity 31: 469-479.

13. Drazen J (1998) Clinical pharmacology of leukotriene receptor antagonists and 5-lipoxygenase inhibitors. American Journal of Respiratory and Critical Care Medicine 157: S233-S237.

14. Huang C, Embrechts MJ, Sukumar N, Breneman CM (2007) Data Fusion and Auto-fusion for Quantitative Structure-Activity Relationship (QSAR). Artificial Neural Networks 4668: 628-637.

15. Mungalpara J, Pandey A, Jain V, Mohan CG (2010) Molecular modelling and QSAR analysis of some structurally diverse N-type calcium channel blockers. Journal of Molecular Modeling 16: 629-644.

16. Habibi-Yangjeh A, Pourbasheer E, Danandeh-Jenagharad M (2009) Application of principal component-genetic algorithm artificialneural network for prediction acidity constant of various nitrogen-containing compounds in water. Monatshefte für Chemie 140: 15-27.

17. Richards ML, Lio SC, Sinha A, Banie H, Thomas RJ, et al. (2006) Substituted 2-phenyl-benzimidazole derivatives: novel compounds that suppress key markers of allergy. European Journal of Medicinal Chemistry 41: 950-969.

18. Vlife MDS software package (2008), version 3.5, supplied by Vlife science technologies Pvt. Ltd, Pune.

19. Golbraikh A, Tropsha A (2002) Predictive QSAR modeling based on diversity sampling of experimental datasets for the training and test set selection. Journal of Computer-Aided Molecular Design 16:357-369.

20. Croux C, Joossens K (2005) Influence of observations on the misclassification probability in quadratic discriminant analysis. Journal of Multivariate Analysis 96: 348-403.

21. Devillers J (1996) Neuronal network in QSAR and drug design. Academic Press, London. 